\title{
Dynamic Balancing and Walking for Real-time 3D Characters
}

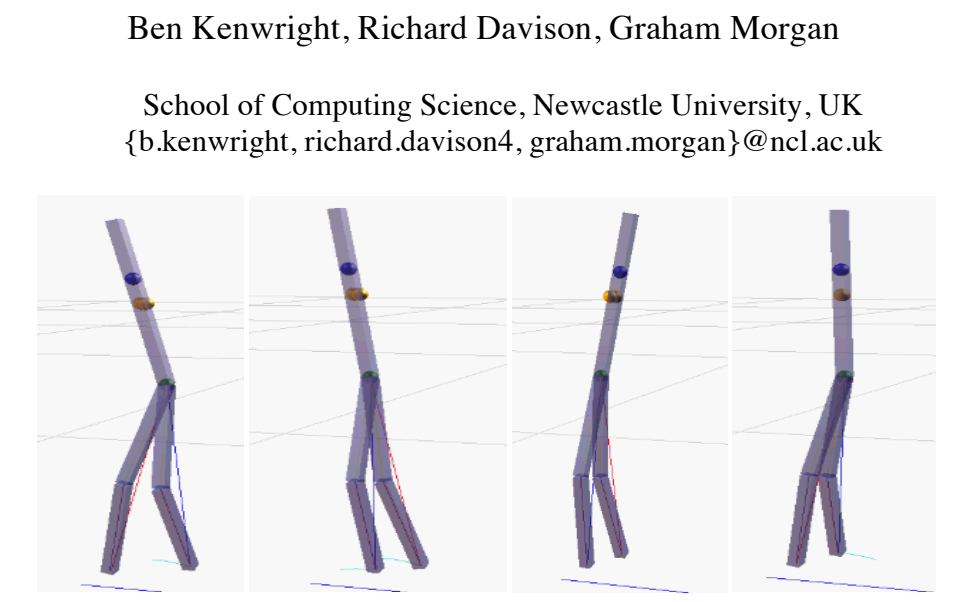

Fig. 1. Predictive stepping and posture correction due to random force disturbances being applied to the body.

\begin{abstract}
This paper describes the real-time modeling of 3D skeletal motion with balancing properties. Our goal is to mimic human responsiveness when external forces are applied to the model. To achieve this we use an inverted pendulum as a basis for achieving a self-balancing model. We demonstrate responsiveness in stepping and posture control via a simplified biped skeletal model using our technique.
\end{abstract}

Keywords: 3D Characters, Balancing, Physics-Based Simulation, Inverted Pendulum, Virtual Pivot Point, Steering.

\section{Introduction}

In many immersive style games there is a need for player-controlled characters to interact with the gaming arena. Such characters provision player representation in a game and usually take human form. As a player understands how humans may act and move given certain physical environments, unexpected or restricted behavior of a player-controlled character is noticeable. Therefore, a challenging research problem is to ensure player-controlled characters are sufficiently expressive to afford the desired level of gameplay while maintaining naturally realistic movements and actions. 
Data driven methods afford player-controlled characters the most natural of movements. Motion capture can ensure key-frames can be used for movement referencing or an artist could create specific animation styles. An enhanced ragdoll model can be used to blend between pre-determined animations to allow for smoothed movement (e.g., [1 - 7]). This data-driven approach looks realistic, but is restrictive in that if the animation is not pre-calculated it may not be possible to achieve. Gameplay, in essence, is restricted when reliance is placed on this technique.

The opposing technique to data driven approaches is to allow a character that is primarily controlled using physics (e.g., [8-14]) (controller approach). In this approach the physical properties of the overall character combine with feedback control principles to generate animation. The major drawback to such approaches is that they do not look as realistic as their data-driven counterparts. One major reason for this is that although the character may achieve the animation goal, it may be achieved in a manner different to that of a human. A further drawback to such methods is the level of computation required to solve non-trivial equations in realtime to calculate movement.

In many instances data driven and controller approaches are combined (e.g., $[39,40,42])$. This has the advantage that fewer pre-determined animation sequences may be required while affording a greater degree of animation. For example, a character falling over on an uneven surface (e.g., stairs) has to be achieved via physics whereas upper limb movement (e.g., reaching) could be achieved via artist directed animation. The popularity of this approach seems to indicate that the purely physics based approach may not suffice on its own (realism is lacking). However, if games are to afford complete freedom of interaction this is the only viable approach.

In this paper we consider a purely controller based approach for realistic human movement that is rendered in real-time. The key to mimicking human movement is not an attempt to recreate the appropriate physical motion alone, but to augment it with a balance controller for posture alignment. Our technique is prompted by work within theoretical robotics where an inverted pendulum is used for such purposes [28]. We demonstrate that our approach favors a human like approach to movement in terms of posture when presented with external forces. Furthermore, we extend the theoretical work described in [28] by moving our model from 2D to 3D and providing a real-time implementation.

\section{Background and Related Work}

In this section we describe a brief overview of the evolution of controller-based approaches to human motion and how such approaches have utilized data driven methods for more realism. We then describe an approach that provisions suitable locomotive control methods that are realistic. Finally, we describe the contribution made by this paper.

\subsection{General Approaches}

Achieving realistic movement of characters is an active area of research in both graphical simulation and robotics. The most basic of requirements is usually that of 
balance when using the controller approach, as without it few other activities are possible. Balancing in the presence of forces has been demonstrated $[14,17,18]$ and more elaborate movements such as walking, running, jumping and cycling have been achieved $[8,12,15,16]$. Further enhancements are to augment the controller with data driven approaches such as motion capture to create more stylistic motion $[19,20]$. The reason for such enhancements is to bring more realism to the motions of the character. A common approach to combining data driven stylistic movement with physics is to allow a controller to maintain balance while upper body movement (which is most noticeable and more intricate) is stylized [20,21].

A controller itself must rely on more than one physics technique if a range of transitional movements is to be accommodated (e.g., $[1,4,10,22])$. SIMBICON [10] is a notable advancement in this area in recent years and demonstrates a biped controller that utilizes a series of poses to shape motion trajectories for flexible locomotion. Considering locomotion techniques in general, irrelevant of stylized interpretation (be it artist or motion capture inspired) a biped must place their feet in a timely manner within a particular surface area to maintain balance. This "swinging motion of legs" has been shown to be achievable via a physics technique that utilizes an inverted pendulum $[23,24]$.

\subsection{Inverted Pendulum}

The linear inverted pendulum (IP) may generate predictive foot placement information for balancing characters in 2D and 3D (e.g., [24, 29]). IP can be used for characters balancing while standing still or during locomotion. The IP motion is constrained to move along an elliptical path. To push the character upright a force is exerted on a character's mass from the center of the foot that is in contact with the ground. As demonstrated in $[30,31]$, the character's mass is concentrated into a single point (center of mass $(\mathrm{CoM})$ ) for the required calculations. To encourage a more natural motion a spring may be associated with the balancing leg [32,33]. This makes the foot trajectory resemble a bouncing ball that mimics, to a visually convincing level, a running motion. This approach is described as a spring loaded inverted pendulum (SLIP).

The IP has been used as a calculation of balance to form the basis on which other aspects of human motion may be constructed [10, 19, 24, 34, 35, 36, 42]. However, as the standard IP model uses a single mass-point in its calculations there is a lack of information present to realistically represent the posture of a character. An approach to correct this issue would be to use a proportional derivative controller [16, 20,37 ] to apply torque to ensure a character's body remains upright. Unfortunately, this corrective torque produces unnaturally movements due to the body not receiving feedback from the characters feet, known as the ground reaction force (GRF). In humans it is this force that causes people to sway and shift as they walk and change direction.

To overcome the problems of unnatural posture control in the IP approach an extended rigid body may be used to represent the trunk (enhanced IP). The hip point would be located at the base of the trunk, the CoM at the mid-point of the trunk and a virtual pivot point (VPP) point located parallel to the extended body and positioned 
above the CoM. This work was initially described in [28] and demonstrates a correlation between natural and simulated GRF. In addition, [38] describes the resultant motion demonstrated in [28] more realistic than IP without extended rigid body.

\subsection{Contribution of Paper}

In this paper we extend the work of the enhanced IP described in [28]. The work in [28] was 2D and only provisioned the calculated expectation of the simulation. In the field of robotics this initial investigatory stage is usual as the end goal is to actually build a robot. However, for video games we want a fully interactive 3D run-time implementation. This is what we achieved, allowing our character model to be exposed to forces and be manipulated by a player. The model itself has no data driven aspects with all foot placing's and body movements created dynamically.

The fundamental contribution of this paper is the demonstration of the enhanced 3D IP as an option for fully interactive realistic human locomotion in realtime without any data driven requirements.

\section{Method}

In this section we describe our method to achieving realistic motion for real-time characters. As we build directly on suggestions found in [28], we describe in more detail IP and then the enhanced version. This allows us to say quite specifically what enhancements we have made. We then describe how balancing, motion and the handling of uneven surfaces (stairs) may be achieved using our approach.

\subsection{Mechanics of IP}

For the purposes of balance control, we consider a human body to exhibit similar qualities to an IP. A human standing on a single leg would be equivalent to an IP assuming the body is represented by a single CoM linked to the ground contact point (center of pressure $(\mathrm{CoP})$ ). In this model we assume the legs to have no mass. This trivializes the calculation significantly while still affording the desired motion. We assume that there is no ground slippage and a single point replaces the foot.

The IP provides a simple technique for predicting where a character should place its feet to remain balanced. The diagram on the left of figure 2 shows how the different elements combine to produce a repeated motion suitable for modeling walking. The springs in the legs represent the fact that we are considering the SLIP approach to improve realism as described in $[32,33]$. 

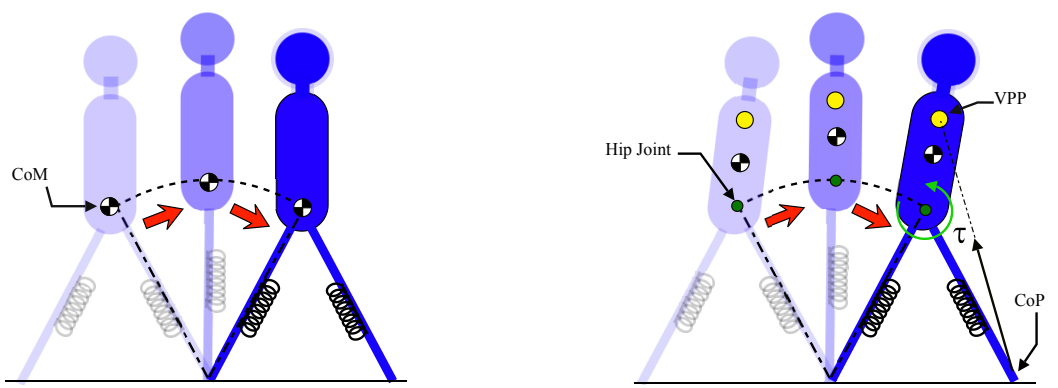

Fig. 2. (Left) Illustrates IP following a basic elliptical trajectory with no body feedback, (Right) the elongated body following a similar ellipsoid-like trajectory during locomotion, with additional torque-body feedback.

\subsection{Mechanics of Enhanced IP}

The enhanced IP model uses all the properties of the basic SLIP model with some important additional features. The enhanced IP is shown on the right of figure 2 . An elongated body is added that represents the trunk [28]. A VPP is added above the hip and parallel to the trunk.

In [28] the author provides a discussion where there is a suggestion that allowing the VPP to move outside the trunk will vary the energy in the model. Varying the energy in such a way would have the effect of speeding up or slowing down the walking motion in characters using the technique in [28]. As [28] dealt only in $2 \mathrm{D}$ speeding up and slowing down was all that could be accomplished. However, we realized that if this approach could be extended to 3D the VPP could ultimately be used for steering the character. Therefore, to pursue this line of research our initial technical change made to [28] was to allow the VPP to move outside the trunk to produce responsive balancing motions.

\subsection{Calculations}

The trunk torque is calculated by projecting the force from the leg (i.e., hip-ground spring) onto the VPP (i.e., ground-VPP) to induce a responsive self-balancing posture.

$$
\begin{gathered}
\widehat{V}_{C}=\frac{(V P P-C o P)}{\|V P P-C o P\|} \\
\widehat{V}_{T R U N K}=\frac{(V P P-C o M)}{\|V P P-C o M\|} \\
\|G R F\|=F_{L} \cdot \hat{V}_{C} \\
G R F=\|G R F\| \cdot \hat{V}_{C}
\end{gathered}
$$




$$
\tau_{\text {hip }}=G R F \times \widehat{V}_{T R U N K}
$$

The directional force from the CoP to the VPP produces the GRF. Using the equations above we extract magnitude and vector to apply a torque to the trunk that feeds back to the hip. This is shown in figure 3 .

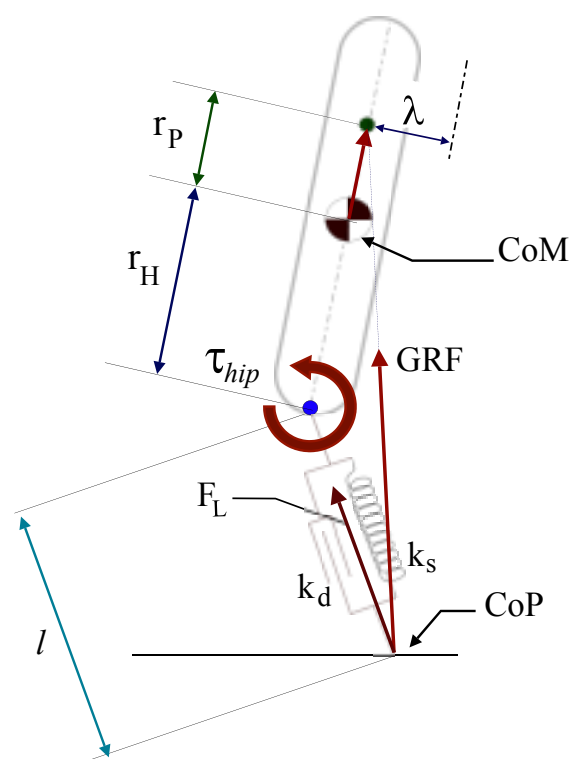

Body Mass: $75 \mathrm{~kg}$

Body Length: $0.9 \mathrm{~m}$

Leg Length $(l): 1.0 \mathrm{~m}$

Step Angle: $\sim 16 \mathrm{deg}$

$\mathrm{r}_{\mathrm{H}}: 0.45 \mathrm{~m}$

$\mathrm{r}_{\mathrm{p}}: 0.18 \mathrm{~m}$

ks: $100000 \mathrm{Nm}^{-1}$

kd: $100 \mathrm{Nm}^{-1}$

Fig. 3. Hip torque calculated using the $\mathrm{CoP}$ and VPP.

\subsection{Managing Stairs and Slopes}

Our approach can be extended to allow a character to use stairs and traverse sloping terrain by offsetting the VPP position proportional to the change in step size. In traditional approaches foot placement would be a costly exercise whereas in our approach it is a byproduct of the movement.
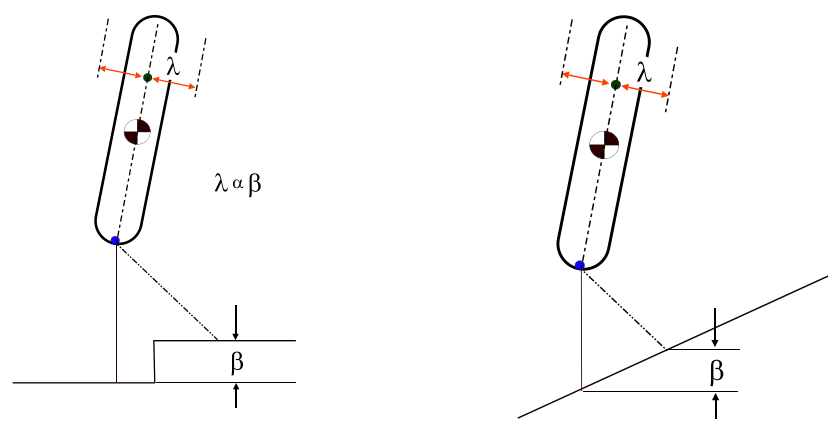
Fig. 4. Walking up stairs/steps or a gradual slope.

$$
\lambda \propto \beta
$$

In (6) $\lambda$ is the offset of the VPP perpendicular to the trunk and $\beta$ is the change in height from the current standing foot placement.

\subsection{Controllable Motion}

When the VPP is shifted to the left or right, the body sways in the appropriate direction (i.e., with the VPP). If the body is moving in a specific direction, the VPP can provide small temporary offsets to maintain, speedup or slowdown a character's overall velocity.

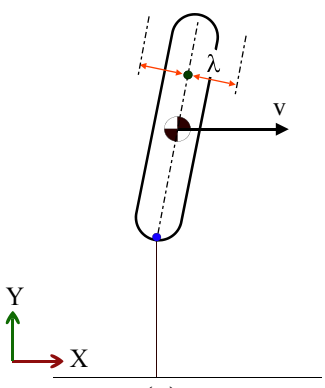

(a)
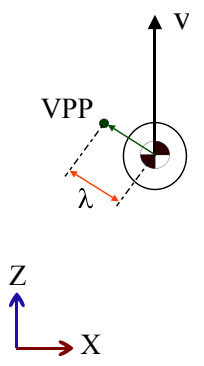

(b)

Fig. 5. Alter the direction of motion by offsetting the VPP (a) Side View (b) Top View.

$$
v_{d} \propto \lambda
$$

We can cause a change in velocity by recognizing that the perpendicular offset relative to the trunk $(\lambda)$ causes a proportional change in the desired speed $\left(v_{d}\right)$. 

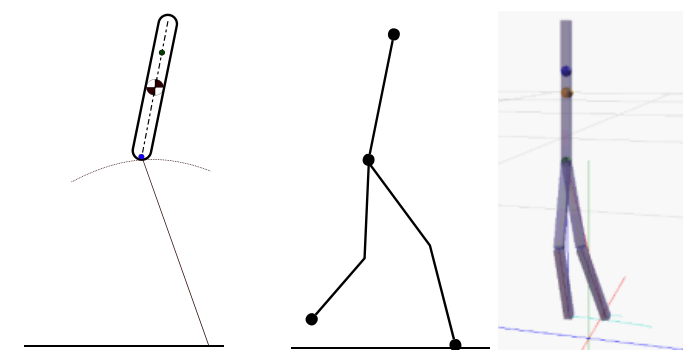

Fig. 6. The enhanced inverted pendulum generates reference motions (feet, hip positions, body orientation) that we use to control our five-link biped.

Possibly the strongest reason for our approach is an ability to handle disturbances such as wind or being pushed. A character will respond naturally to the disturbing forces and attempt to take corrective steps to remain balanced. The corrective stepping automatically provides feedback to the trunk orientation to reflect these dynamic changes.

\section{Results}

In this section we present preliminary results for our method. Our method is used to control a five-link biped model. We consider steering, speed control and balancing in the presence of pushing. Diagrams are provided that show the different snapshots of movement over time. A YouTube video of our experiments may be viewed at [41]

\subsection{Steering and Speed}

A turn angle less than 10 degrees kept our locomotion natural, realistic and stable. Spline paths where created for a character to follow and enabled us to evaluate the applicability of using the VPP for steering.

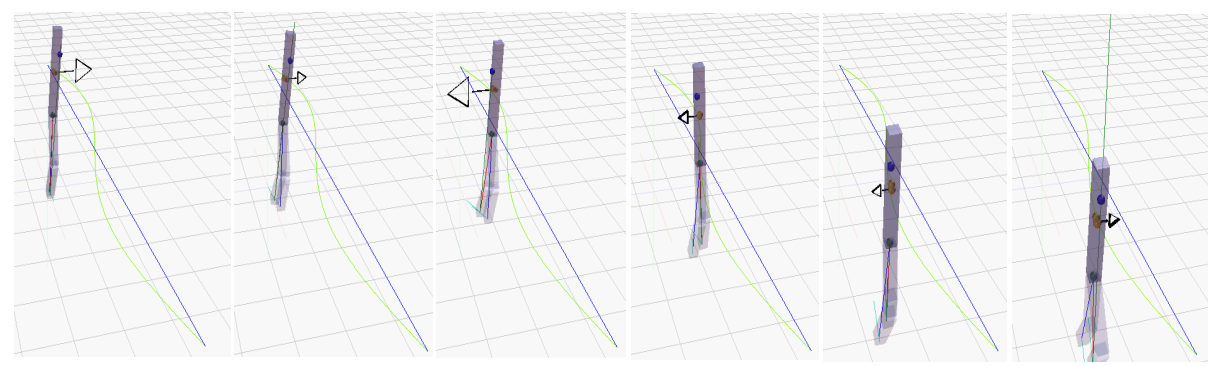

Fig. 7. Alter the VPP-offset to control the steering locomotion (Arrow in the figure indicates the desired steering direction).

The model was able to produce various types of animation and movement. These included: standing still (balancing), starting to walk, taking 2-5 steps then stopping, 
walking following various paths. Changing the VPP enabled us to create varying gaits dynamically (slow/fast walking).

\subsection{Robustness to Pushes}

We applied external disturbances to determine the robustness of our model (an ability to balance realistically). We set our model walking at a stable gait then randomly applied forces between $50 \mathrm{~N}$ to $100 \mathrm{~N}$ for 0.1 to 0.4 seconds at the CoM. The model passed the test if the body remained upright. Altering body mass and leg parameters affects the robustness of the model. This is a desired outcome, as eventually we would like to model a variety of human body types to gain realistic behaviors within crowds.

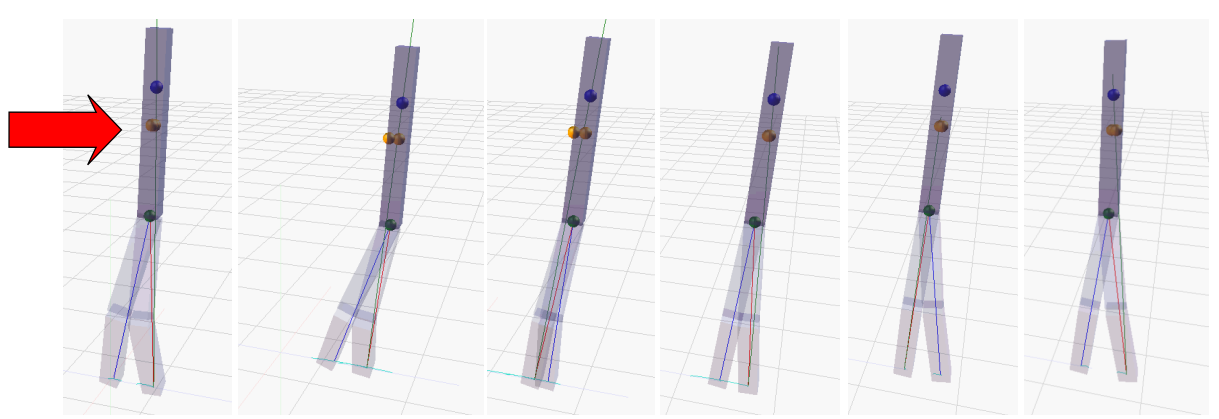

Fig. 8. Incremental screenshots of the biped model responding to a $100 \mathrm{~N}$ push force being applied to the CoM for $0.1 \mathrm{~s}$.

\section{Conclusions and Future Work}

We have described an approach for modeling mid to lower body 3D human movement in real-time. We require no data driven (e.g., key frame) elements to achieve such movement and our approach exhibits robust self-balancing properties. Furthermore, the enhanced IP used in our model also introduces movement that resembles human motion by considering the trunk of the body and hip together in posture calculations. This is the first time a real-time 3D model has made use of this technique. Significantly, we have described a technique that is not only computationally constrained, making it suitable for use in video games, but also exhibits motion that is human like without any artist or key-frame intervention.

Work will continue with the enhancement of the model to include additional body parts (e.g., arms, head, hands, feet). In addition, we will carry out comparisons to determine how well our model captures human type movement in a detailed manner as in [43]. Eventually we will create a model that exhibits sufficient realism in most instances of movement but also allows data driven approaches to superimpose desired styles. This is a must for video games as replicating realistic human movement is not the end goal because style must also be conveyed within a gaming context (e.g., angry, sad, panic). 


\section{References}

[1] A. Sulejmanpašić and J. Popović, "Adaptation of performed ballistic motion," ACM Transactions on Graphics, vol. 24, Jan. 2005, pp. 165-179.

[2] C.K. Liu, A. Hertzmann, and Z. Popović, "Learning physics-based motion style with nonlinear inverse optimization," ACM Transactions on Graphics, vol. 24, Jul. 2005, p. 1071.

[3] A. Safonova, J.K. Hodgins, and N.S. Pollard, "Synthesizing physically realistic human motion in low-dimensional, behavior-specific spaces," ACM Transactions on Graphics, vol. 23, Aug. 2004, p. 514.

[4] A.C. Fang and N.S. Pollard, "Efficient synthesis of physically valid human motion," ACM Transactions on Graphics, vol. 22, Jul. 2003, p. 417.

[5] M.J. Mandel, "Versatile and Interactive Virtual Humans : Hybrid use of DataDriven and Dynamics-Based Motion Synthesis," Simulation, 2004.

[6] P. Wrotek, O.C. Jenkins, M. McGuire, "Dynamo: Dynamic , Data-driven Character Control with Adjustable Balance," ACM Sandbox Symposium on Video Games, 2006.

[7] J. Lee, J. Chai, P.S. a Reitsma, J.K. Hodgins, and N.S. Pollard, "Interactive control of avatars animated with human motion data," Proceedings of the 29th annual conference on Computer graphics and interactive techniques SIGGRAPH '02, 2002, p. 491.

[8] J.K. Hodgins, W.L. Wooten, D.C. Brogan, and J.F. O’Brien, “Animating human athletics," Proceedings of the 22nd annual conference on Computer graphics and interactive techniques - SIGGRAPH '95, 1995, pp. 71-78.

[9] D. Sharon and M. van de Panne, "Synthesis of Controllers for Stylized Planar Bipedal Walking," Proceedings of the 2005 IEEE International Conference on Robotics and Automation, pp. 2387-2392.

[10] K. Yin, "SIMBICON : Simple Biped Locomotion Control," Control, vol. 26, 2007, pp. 1-10.

[11] K.W. Sok, M. Kim, and J. Lee, "Simulating Biped Behaviors from Human Motion Data," Database, vol. 26, 2007.

[12] J.M. Wang, D.J. Fleet, and A. Hertzmann, "Optimizing walking controllers," ACM Transactions on Graphics, vol. 28, Dec. 2009, p. 1.

[13] M.D. Lasa, "Feature-Based Locomotion Controllers," Computer Graphics Forum, 2010.

[14] A.H. Adiwahono, C.-M. Chew, W. Huang, and Y. Zheng, "Push recovery controller for bipedal robot walking," 2009 IEEE/ASME International Conference on Advanced Intelligent Mechatronics, Jul. 2009, pp. 162-167.

[15] J. Pratt and G. Pratt, "Exploiting natural dynamics in the control of a 3d bipedal walking simulation," Proceedings of the International Conference on Climbing and Walking Robots, Citeseer, 1999, p. 797-807.

[16] M.H. Raibert, Legged Robots That Balance, MIT Press, 1986.

[17] B.J. Stephens and C.G. Atkeson, "Push Recovery by Stepping for Humanoid Robots with Force Controlled Joints," Primus.

[18] B. Stephens, "Humanoid push recovery," 2007 7th IEEE-RAS International Conference on Humanoid Robots, Nov. 2007, pp. 589-595. 
[19] Y. Abe and M. Silva, "Multiobjective Control with Frictional Contacts," Computing, 2007.

[20] V.B. Zordan and J.K. Hodgins, "Motion capture-driven simulations that hit and react," Proceedings of the 2002 ACM SIGGRAPH/Eurographics symposium on Computer animation - SCA '02, New York, New York, USA: ACM Press, 2002, p. 89.

[21] S. Nakaoka, a Nakazawa, K. Yokoi, H. Hirukawa, and K. Ikeuchi, "Generating whole body motions for a biped humanoid robot from captured human dances," 2003 IEEE International Conference on Robotics and Automation (Cat. No.03CH37422), pp. 3905-3910.

[22] P. Faloutsos, M. van de Panne, and D. Terzopoulos, "The virtual stuntman: dynamic characters with a repertoire of autonomous motor skills," Computers \& Graphics, vol. 25, Dec. 2001, pp. 933-953.

[23] J.-Y. Kim, I.-W. Park, and J.-H. Oh, "Walking Control Algorithm of Biped Humanoid Robot on Uneven and Inclined Floor," Journal of Intelligent and Robotic Systems, vol. 48, Jan. 2007, pp. 457-484.

[24] Y.-Y. Tsai, W.-C. Lin, K.B. Cheng, J. Lee, and T.-Y. Lee, "Real-time physics-based 3D biped character animation using an inverted pendulum model.," IEEE transactions on visualization and computer graphics, vol. 16, 2010, pp. 325-37.

[25] J.K. Hodgins and N.S. Pollard, "Adapting simulated behaviors for new characters," Proceedings of the 24th annual conference on Computer graphics and interactive techniques - SIGGRAPH '97, 1997, pp. 153-162.

[26] K. Yin, S. Coros, P. Beaudoin, and M. van de Panne, "Continuation methods for adapting simulated skills," ACM Transactions on Graphics, vol. 27, Aug. 2008, p. 1 .

[27] S. Coros, P. Beaudoin, K.K. Yin, and M. van de Pann, "Synthesis of constrained walking skills," ACM Transactions on Graphics, vol. 27, Dec. 2008 , p. 1 .

[28] H. Maus, J. Rummel, and A. Seyfarth, "Stable Upright Walking and Running using a simple Pendulum based Control Scheme," Spring, 2008, pp. 4-10.

[29] S. Kajita, F. Kanehiro, K. Kaneko, K. Yokoi, and H. Hirukawa, "The 3D linear inverted pendulum mode: a simple modeling for a biped walking pattern generation," Proceedings 2001 IEEE/RSJ International Conference on Intelligent Robots and Systems. Expanding the Societal Role of Robotics in the the Next Millennium (Cat. No.01CH37180), 2001, pp. 239-246.

[30] S. Kajita and K. Tan, "Study of Dynamic Biped Locomotion on Rugged Terrain - Derivation and Application of the Linear Inverted Pendulum Mode ," Mechanical Engineering, 1991, pp. 1405-1411.

[31] S. Kajita and K. Tani, "Experimental study of biped dynamic walking in the linear inverted pendulum mode," Proceedings of 1995 IEEE International Conference on Robotics and Automation, 1965, pp. 2885-2891.

[32] R. Blickhan and R.J. Full, "Similarity in multilegged locomotion: Bouncing like a monopode," Journal of Comparative Physiology A, vol. 173, Nov. 1993. 
[33] R. Full and C.T. Farley, "Musculoskeletal dynamics in rhythmic systems: a comparative approach to legged locomotion.," Biomechanics and Neural Control of Posture and Movement. Springer-Verlag, New York, 2000.

[34] M.H. Raibert and J.K. Hodgins, "Animation of dynamic legged locomotion," ACM SIGGRAPH Computer Graphics, vol. 25, Jul. 1991, pp. 349-358.

[35] J. Pratt, "Virtual Model Control: An Intuitive Approach for Bipedal Locomotion," The International Journal of Robotics Research, vol. 20, Feb. 2001, pp. 129-143.

[36] S. Kajita, F. Kanehiro, K. Kaneko, K. Fujiwara, K. Harada, K. Yokoi, and H. Hirukawa, "Biped walking pattern generation by using preview control of zero-moment point," 2003 IEEE International Conference on Robotics and Automation (Cat. No.03CH37422), 2003, pp. 1620-1626.

[37] N. Neville, M. Buehler, and I. Sharf, "A bipedal running robot with one actuator per leg," Proceedings 2006 IEEE International Conference on Robotics and Automation, 2006. ICRA 2006., 2006, pp. 848-853.

[38] J. Rummel and A. Seyfarth, "Passive stabilization of the trunk in walking," Autonomous Robots, 2010, pp. 127-136.

[39] Victor Zordan, Adriano Macchietto, Jose Medina, Marc Soriano, and ChunChih Wu. 2007. Interactive dynamic response for games. In Proceedings of the 2007 ACM SIGGRAPH symposium on Video games (Sandbox '07). ACM, New York, NY, USA, 9-14. DOI=10.1145/1274940.1274944 http://doi.acm.org/10.1145/1274940.1274944

[40] Victor Brian Zordan, Anna Majkowska, Bill Chiu, and Matthew Fast. 2005. Dynamic response for motion capture animation. In ACM SIGGRAPH 2005 Papers (SIGGRAPH '05), Markus Gross (Ed.). ACM, New York, NY, USA, 697-701. DOI $=10.1145 / 1186822.1073249$ http://doi.acm.org/10.1145/1186822.1073249

[41] [Online] Available: http://www.youtube.com/watch?v=jLIMSD8jcBc

[42] S. Coros, P. Beaudoin, and M. van de Panne. Generalized biped walking control. ACM Transactions on Graphics, 29(4):Article 130, 2010.

[43] Igor Mordatch, Martin de Lasa, and Aaron Hertzmann. 2010. Robust physicsbased locomotion using low-dimensional planning. In ACM SIGGRAPH 2010 papers (SIGGRAPH '10), Hugues Hoppe (Ed.). ACM, New York, NY, USA, , Article 71,8 pages. DOI $=10.1145 / 1833349.1778808$

http://doi.acm.org/10.1145/1833349.1778808 